TRANSACTIONS OF THE

AMERICAN MATHEMATICAL SOCIETY

Volume 365, Number 12, December 2013, Pages 6367-6383

S 0002-9947(2013)05851-5

Article electronically published on June 3, 2013

\title{
DETERMINISTIC APPROXIMATIONS OF RANDOM REFLECTORS
}

\author{
OMER ANGEL, KRZYSZTOF BURDZY, AND SCOTT SHEFFIELD
}

\begin{abstract}
Within classical optics, one may add microscopic "roughness" to a macroscopically flat mirror so that parallel rays of a given angle are reflected at different outgoing angles. Taking the limit (as the roughness becomes increasingly microscopic) one obtains a flat surface that reflects randomly, i.e., the transition from incoming to outgoing ray is described by a probability kernel (whose form depends on the nature of the microscopic roughness).

We consider two-dimensional optics (a.k.a. billiards) and show that every random reflector on a line that satisfies a necessary measure-preservation condition (well established in the theory of billiards) can be approximated by deterministic reflectors in this way.
\end{abstract}

\section{INTRODUCTION}

This article addresses the question of which random reflectors can be approximated by piecewise smooth surfaces that reflect light according to the classical rule of specular reflection which says that the angle of reflection is equal to the angle of incidence. One of the basic results on billiards says that a certain measure on the space of pairs consisting of location and angle of reflection is preserved by every reflecting surface (see [17, Thm. 3.1] or [3, Lemma 2.35]). Our main result, Theorem 2.3. shows that, except for this universal restriction that applies to all reflecting surfaces, one can approximate in a weak sense every random reflector by a sequence of specularly (deterministically) reflecting surfaces.

A special case of this theorem is that one can approximate every deterministic reflector that preserves the appropriate measure, including, for example, the reflector that reverses the direction of each incoming ray. To see how counterintuitive this is, imagine a higher dimensional analog of this reflector: a mirror that appears entirely the color of the observer's eyeball, because the only light rays traveling from the mirror to the eyeball are those that bounced off of the eyeball before reaching the mirror. This striking effect is implemented in practice with limited accuracy (for theoretical and practical reasons) in "retroreflectors" (see [18]) and reflective paint (see [19]).

There are several sources of inspiration for our project. The article 1 studies reflected Brownian motion with inert drift. In a follow up project, Z.-Q. Chen and the second author plan to study the limiting situation when the diffusion coefficient

Received by the editors March 4, 2012 and, in revised form, April 7, 2012 and April 9, 2012. 2010 Mathematics Subject Classification. Primary 37D50.

The first author's research was supported in part by NSERC and by the Sloan Foundation. The second author's research was supported in part by NSF Grant DMS-0906743 and by grant N N201 397137, MNiSW, Poland. The third author's research was supported in part by NSF Grant DMS 0645585. 
of reflected Brownian motion goes to zero. It appears that in the limit, the particle will move along straight lines with a random angle of reflection.

The physics literature on random reflections is quite rich. We will not review it here; an excellent review can be found, for example, in [5].

Some early mathematical articles that considered random reflections were 14, 9, and more recent ones include [4, 5, 6, 7, 8, 11, 12, 15, 16. Many of these articles are concerned with the so-called Knudsen reflection law. Knudsen proposed the cosine reflection law, in which the direction of the reflected molecule has a rotation-invariant distribution around the surface normal, with density proportional to the cosine of the angle with the normal, independent of the incidence direction. Knudsen's law is a model for gas dynamics. The same law is known as Lambert's cosine law in optics (see [2], pp. 147-148 or [13, Chap. 6).

On the technical side, our results seem to be related, at least at the intuitive level, to the "digital sundial" theorem proved by Falconer (see [10, Thm. 6.9). Roughly speaking, Falconer's theorem says that there exists a set with prescribed projections in almost all directions.

Although our article is close to the literature on billiards at the technical level, we will use the language of optics because our model is much closer to this circle of ideas at the intuitive level.

We will state our results in a rigorous way in Section 2 Section 3 contains short proofs of the most elementary results. The proof of our main result, Theorem 2.3, is a multistage construction presented in Sections 45 .

\section{The MAin RESUlt, CONJeCtures AND OPEn PROBlems}

For a very detailed and careful presentation of the billiards model in the plane, see Chap. 2 of 3 . We will be concerned with mirrors (walls of billiard tables) of very special shape. They are supposed to model macroscopically flat but rough reflecting surfaces. The paper of Feres 11] contains a rigorous mathematical presentation of this physical phenomenon and detailed analysis of its fundamental properties. Our setup is slightly different from that in [11.

Consider the following assumptions about a planar set $M$. These conditions contain, among other things, Assumptions A1-A4 from [3, Sections 2.1 and 2.4].

(M1) $M \subset\left\{\left(x_{1}, x_{2}\right) \in \mathbb{R}^{2}: x_{2}<0\right\}$.

(M2) For every $k<\infty$, the set $\left\{\left(x_{1}, x_{2}\right) \in M:-k \leq x_{1} \leq k\right\}$ is the union of a finite number of compact $C^{3}$ curves $\Gamma_{j}$.

(M3) The curves $\Gamma_{j}$ intersect only at their endpoints. Each curve either is a line segment or has non-vanishing curvature of one sign (it has no inflection points). The curves do not form cusps at the intersection points; that is, the curves form an angle different from 0 at the point where they meet (if there is such a point).

We will say that $M \in \mathcal{M}_{1}$ if $M$ satisfies (M1)-(M3).

Let $D=\mathbb{R} \times(-\pi, 0)$ and define a $\sigma$-finite measure $\Lambda$ on $D$ by $\Lambda(d x, d \alpha)=$ $-d x \sin \alpha d \alpha$.

A light ray can be represented as $(x, \alpha)=(x(t), \alpha(t))$, where $x(t)$ is the location of the light ray at time $t$ and $\alpha(t) \in(-\pi, \pi]$ is the angle between the direction of the light ray and the positive horizontal half-axis, measured in the counterclockwise direction from the half-axis. Time will play no role in our arguments, so it will be suppressed in the notation most of the time. We will always assume that light rays reflect from surfaces comprising $M \in \mathcal{M}_{1}$ according to the rule of specular 
reflection; that is, the angle of incidence is equal to the angle of reflection, for every reflection.

Let $L_{*}:=\left\{\left(x_{1}, x_{2}\right) \in \mathbb{R}^{2}: x_{2}=0\right\}$. It will be convenient to identify $L_{*}$ with $\mathbb{R}$; for example, we will consider $\Lambda$ to be a measure on $L_{*} \times(-\pi, 0)$. Consider the following natural condition.

(M4) Suppose that $M \in \mathcal{M}_{1}$ and for $\Lambda$-almost all $(x, \alpha) \in D$, a light ray starting from $(x, \alpha)$ and reflecting from surfaces comprising $M$ will return to $L_{*}$ after a finite number of reflections.

Condition (M4) is far from trivial; see, for example, Sec. 2.4 in [3] on accumulations of collisions. Moreover, some light rays reflecting from some mirror sets $M \in \mathcal{M}_{1}$ will not return to $L_{*}$. We will show that (M4) holds for a large class of sets $M$.

(M5) Suppose that $M \in \mathcal{M}_{1}$. Let $\left\{A_{k}^{\prime}\right\}_{k \geq 1}$ be the family of all connected components of the open set $\left\{\left(x_{1}, x_{2}\right) \in \mathbb{R}^{2} \backslash M: x_{2}<0\right\}$. There exists a subfamily $\left\{A_{k}\right\}_{k \geq 1}$ of $\left\{A_{k}^{\prime}\right\}_{k \geq 1}$ (that is, every set $A_{k}$ is equal to some set $A_{j}^{\prime}$ ), such that every set $A_{k}$ is bounded, $L_{*} \subset \bigcup_{k \geq 1} \partial A_{k}$, and the set $\left\{\partial A_{j} \cap \partial A_{k} \cap L_{*}, j, k \geq 1, j \neq k\right\}$ has no accumulation points in $L_{*}$.

We will say that $M \in \mathcal{M}_{2}$ if $M \in \mathcal{M}_{1}$ and it satisfies (M4).

Proposition 2.1. If $M \in \mathcal{M}_{1}$ satisfies (M5), then it satisfies (M4) and, therefore, $M \in \mathcal{M}_{2}$.

Consider some $M \in \mathcal{M}_{2}$. Suppose that a light ray starts from $\left(x_{0}, \alpha_{0}\right)$ with $x_{0} \in L_{*}$ and $\alpha_{0} \in(-\pi, 0)$ at time 0 , reflects from surfaces of $M$ and returns to $L_{*}$ at a time $t$, i.e., $(x(t-), \alpha(t-))=\left(x_{1}, \beta^{\prime}\right), x_{1} \in L_{*}$, and $t>0$ is the smallest time with this property. Let $\beta=\beta^{\prime}-\pi$. This defines a mapping $K: D \rightarrow D$, given by $K(x, \alpha)=(y, \beta)$. Clearly, $K$ depends on $M$.

We will write $\mathbb{P}(x, \alpha ; d y, d \beta)$ to denote a Markov transition kernel on $D$; that is, for fixed $(x, \alpha) \in D, \mathbb{P}(x, \alpha ; d y, d \beta)$ is a probability measure on $D$. We assume that $\mathbb{P}$ satisfies the usual measurability conditions in all variables.

We will use $\delta_{x}(y)$ to denote Dirac's delta function. Recall the transformation $K$ and let $\mathbb{P}_{K}$ be defined by $\mathbb{P}_{K}(x, \alpha ; d y, d \beta)=\delta_{K(x, \alpha)}(y, \beta) d y d \beta$. In other words, $\mathbb{P}_{K}$ represents a deterministic Markov kernel, with the atom at $K(x, \alpha)$.

If $\mu_{n}, n \geq 1$, and $\mu_{\infty}$ are non-negative $\sigma$-finite measures on some measurable space $\Gamma$, then we will say that $\mu_{n}$ converge weakly to $\mu_{\infty}$ if there exists a sequence of sets $\Gamma_{j}, j \geq 1$, such that $\bigcup_{j \geq 1} \Gamma_{j}=\Gamma, \mu_{n}\left(\Gamma_{j}\right)<\infty, \mu_{\infty}\left(\Gamma_{j}\right)<\infty$ for all $n$ and $j$, and for every fixed $j$, the sequence $\mu_{n}\left(\Gamma_{j}\right)$ converges weakly to $\mu_{\infty}\left(\Gamma_{j}\right)$.

Theorem 2.2. (i) Consider the transformation $K: D \rightarrow D$ corresponding to any $M \in \mathcal{M}_{2}$. The transformation $K$ preserves measure $\Lambda$; that is, for any $A \subset D$ with $\Lambda(A)<\infty$, we have $\Lambda\left(K^{-1}(A)\right)=\Lambda(A)$. Moreover, $K$ is "time reversible" in the sense that if $K\left(A_{1}\right)=A_{2}$, then $K\left(A_{2}\right)=A_{1}$.

(ii) Suppose that for some sequence of sets $M_{n} \in \mathcal{M}_{2}$, corresponding transformations $K_{n}$, and some Markov transition kernels $\mathbb{P}(x, \alpha ; d y, d \beta)$, we have

$$
\Lambda(d x, d \alpha) \mathbb{P}_{K_{n}}(x, \alpha ; d y, d \beta) \rightarrow \Lambda(d x, d \alpha) \mathbb{P}(x, \alpha ; d y, d \beta)
$$

in the sense of weak convergence on $D^{2}$ as $n \rightarrow \infty$. Then $\mathbb{P}$ is symmetric with respect to $\Lambda$ in the sense that for any smooth functions $f$ and $g$ on $D$ with compact 
support, we have

$$
\begin{array}{rl}
\int_{D^{2}} & f(y, \beta) \mathbb{P}(x, \alpha ; d y, d \beta) g(x, \alpha) \Lambda(d x, d \alpha) \\
\quad=\int_{D^{2}} g(y, \beta) \mathbb{P}(x, \alpha ; d y, d \beta) f(x, \alpha) \Lambda(d x, d \alpha) .
\end{array}
$$

In particular, $\Lambda$ is invariant in the sense that

$$
\int_{D^{2}} f(y, \beta) \mathbb{P}(x, \alpha ; d y, d \beta) \Lambda(d x, d \alpha)=\int_{D} f(x, \alpha) \Lambda(d x, d \alpha) .
$$

See [11, Sect. 4] for a similar result stated in a slightly different setting. The first part of the theorem says that all specular reflections are time reversible and preserve a certain measure. For this reason, $\Lambda$ is known as the invariant measure for the collision map in the theory of billiards (see [3, Sec. 2.12). This is related to Lambert's cosine law in optics (see [2, pp. 147-148 or [13, Chap. 6), also known as Knudsen's cosine reflection law in the context of gas dynamics (see [5]). The second part shows that this condition can be interpreted as symmetry for a Markov kernel (see (2.2)). This symmetry is preserved under weak limits of Markov kernels. The next theorem, which is our main result, says that the symmetry of the Markov kernel expressed in (2.2) is the only condition on a Markov kernel $\mathbb{P}$ necessary for the existence of deterministic approximations of random reflections represented by $\mathbb{P}$.

Recall that $\delta_{x}(y)$ denotes Dirac's delta function. Suppose that the probability kernel $\mathbb{P}$ in Theorem 2.2 (ii) satisfies $\mathbb{P}(x, \alpha ; d y, d \beta)=\delta_{x}(y) d y \widetilde{\mathbb{P}}(x, \alpha ; d \beta)$ for some $\widetilde{\mathbb{P}}$. Heuristically, this means that the light ray released at $x$ is instantaneously reflected from a mirror located infinitesimally close to $L_{*}$. Then (2.2) and (2.3) imply that for all smooth bounded functions $f$ and $g$ on $(-\pi, 0)$, and almost all $x$,

$$
\int_{(-\pi, 0)^{2}} f(\beta) \widetilde{\mathbb{P}}(x, \alpha ; d \beta) g(\alpha) \sin \alpha d \alpha=\int_{(-\pi, 0)^{2}} g(\beta) \widetilde{\mathbb{P}}(x, \alpha ; d \beta) f(\alpha) \sin \alpha d \alpha
$$

and

$$
\int_{(-\pi, 0)^{2}} f(\beta) \widetilde{\mathbb{P}}(x, \alpha ; d \beta) \sin \alpha d \alpha=\int_{(-\pi, 0)} f(\alpha) \sin \alpha d \alpha .
$$

Theorem 2.3. Suppose that $\mathbb{P}(x, \alpha ; d y, d \beta)=\delta_{x}(y) d y \widetilde{\mathbb{P}}(x, \alpha ; d \beta)$, where $\widetilde{\mathbb{P}}$ satisfies (2.4). Then there exists a sequence of sets $M_{n} \in \mathcal{M}_{2}$ and corresponding transformations $K_{n}$ such that

$$
\Lambda(d x, d \alpha) \mathbb{P}_{K_{n}}(x, \alpha ; d y, d \beta) \rightarrow \Lambda(d x, d \alpha) \mathbb{P}(x, \alpha ; d y, d \beta)
$$

weakly on $D^{2}$ as $n \rightarrow \infty$. Moreover, $M_{n}$ can be chosen in such a way that

(a) $M_{n} \subset\left\{\left(x_{1}, x_{2}\right):-1 / n<x_{2}<0\right\}$ and

(b) for every $\varepsilon>0$ there exists $D_{\varepsilon} \subset D$ with $\Lambda\left(D \backslash D_{\varepsilon}\right)<\varepsilon$ such that all rays starting in $D_{\varepsilon}$ reflect from $M_{n}$ exactly twice before returning to $L_{*}$.

Remark 2.4. The sets $M_{n}$ that we construct in the proof of Theorem 2.3 are not connected. We believe that the following conjecture can be proved using an iteration of the construction used in the proof of Theorem 2.3 .

Conjecture 2.5. One can construct sets $M_{n}$ so that they satisfy Theorem 2.3 except for (b); they are connected and every light ray reflects from $M_{n}$ four times, except for a set of rays of $\Lambda$ measure $1 / n$. 
The following problem is inspired by the "digital sundial" theorem of Falconer (see [10, Thm. 6.9).

Problem 2.6. Is it possible to construct sets $M_{n}$ so that they satisfy Theorem 2.3 and every light ray reflects from $M_{n}$ only once, except for a set of rays of $\Lambda$ measure $1 / n ?$

We believe that an analogue of Theorem 2.3 holds in higher dimensions.

\section{Deterministic REFLECTIONS}

Proof of Proposition 2.1. Assumptions A1-A4 from [3. Sections 2.1 and 2.4] are built into (M1)-(M3). Hence, there are no accumulation points for reflections from $M$, by the results in [3, Sect. 2.4].

The remaining part of the proof is based on [17, Thm. 3.1 and Sect. 7.1]. We will only outline the main steps. Fix some $k$ and consider $A_{k}$ to be a bounded billiard table. We define the "billiard ball map" $T$ as follows. Let $v(x)$ be a continuous unit tangent vector field on $\partial A_{k}$. For a light ray starting from a point $x \in \partial A_{k}$, let $\alpha$ be the angle between its direction and $v(x)$. The light ray will hit $\partial A_{k}$ at a point $y \in \partial A_{k}$. Let $\beta$ be the angle formed by the direction of the light ray just after reflection at $y$ and $v(y)$. Then we let $T(x, \alpha)=(y, \beta)$. Let $d x$ represent the arc length measure on $\partial A_{k}$. By [17, Thm. 3.1], $T$ preserves the measure $d x \sin \alpha d \alpha$ on $\partial A_{k} \times(0, \pi)$. Poincaré's Recurrence Theorem (see [17, Thm. 7.4]) shows that $(d x d \alpha)$-almost all rays starting at $\left(\partial A_{k} \cap L_{*}\right) \times(0, \pi)$ return to $\left(\partial A_{k} \cap L_{*}\right) \times(0, \pi)$ after a finite number of reflections; see [17, page 116] for more details.

Proof of Theorem 2.2. (i) The claim that $\Lambda\left(K^{-1}(A)\right)=\Lambda(A)$ is a special case of a well-known theorem; see [17, Thm. 3.1] or [3, Lemma 2.35]. The fact that the light reflection process is time reversible implies that if $K\left(A_{1}\right)=A_{2}$, then $K\left(A_{2}\right)=A_{1}$.

(ii) Suppose that $M_{n} \in \mathcal{M}_{2}$ and $K_{n}$ is the corresponding transformation. Consider some sets $A_{1}, A_{2} \in D$ and let $f(x, \alpha)=\mathbf{1}_{A_{1}}(x, \alpha)$ and $g(x, \alpha)=\mathbf{1}_{A_{2}}(x, \alpha)$. Then, using part (i),

$$
\begin{aligned}
& \int_{D^{2}} f(y, \beta) \mathbb{P}_{K_{n}}(x, \alpha ; d y, d \beta) g(x, \alpha) \Lambda(d x, d \alpha) \\
& =\int_{D^{2}} \mathbf{1}_{A_{1}}(y, \beta) \delta_{K_{n}(x, \alpha)}(d y, d \beta) \mathbf{1}_{A_{2}}(x, \alpha) \Lambda(d x, d \alpha) \\
& =\int_{D^{2}} \mathbf{1}_{K_{n}^{-1}\left(A_{1}\right)}(x, \alpha) \mathbf{1}_{A_{2}}(x, \alpha) \Lambda(d x, d \alpha) \\
& =\Lambda\left(K_{n}^{-1}\left(A_{1}\right) \cap A_{2}\right)=\Lambda\left(K_{n}^{-1}\left(A_{2}\right) \cap A_{1}\right) .
\end{aligned}
$$

For the same reason,

so

$$
\int_{D^{2}} g(y, \beta) \mathbb{P}_{K_{n}}(x, \alpha ; d y, d \beta) f(x, \alpha) \Lambda(d x, d \alpha)=\Lambda\left(K_{n}^{-1}\left(A_{2}\right) \cap A_{1}\right),
$$

$$
\begin{array}{rl}
\int_{D^{2}} & f(y, \beta) \mathbb{P}_{K_{n}}(x, \alpha ; d y, d \beta) g(x, \alpha) \Lambda(d x, d \alpha) \\
& =\int_{D^{2}} g(y, \beta) \mathbb{P}_{K_{n}}(x, \alpha ; d y, d \beta) f(x, \alpha) \Lambda(d x, d \alpha) .
\end{array}
$$

A standard argument based on finite linear combinations of step functions and bounded convergence shows that (2.2) holds for smooth $f$ and $g$ with compact 
support and $\mathbb{P}$ of the form $\mathbb{P}_{K_{n}}$. This and weak convergence imply that (2.2) also holds for Markov kernels $\mathbb{P}$ that satisfy (2.1). We obtain (2.3) from (2.2) by the monotone convergence theorem applied to a sequence $g_{n} \uparrow 1$ as $n \rightarrow \infty$.

\section{TRANSPOSITION REFLECTOR}

This section is devoted to the construction of a set of mirrors that transpose thin bundles of light of appropriate angular width. The first challenge is to guide bundles of light rays so that only an arbitrarily small amount of light is scattered in an unaccounted for way. The second challenge (easier to present in a rigorous way than the first one) is to place various sets of mirrors so that they do not interfere with each other.

Let $\bar{A}$ denote the closure of a set $A$.

Definition 4.1. We will call $K: D \rightarrow D$ a simple symmetric function if there exists a countable family of rectangles $Q_{k}=\left(x_{1}^{k}, x_{2}^{k}\right) \times\left(\alpha_{1}^{k}, \alpha_{2}^{k}\right) \subset D, k \geq 1$, such that

(i) $Q_{k} \cap Q_{j}=\emptyset$ for $k \neq j$,

(ii) $\bigcup_{k \geq 1} \bar{Q}_{k}=D$,

(iii) for any $j, k$, either $\left(x_{1}^{k}, x_{2}^{k}\right)=\left(x_{1}^{j}, x_{2}^{j}\right)$ or $\left(x_{1}^{k}, x_{2}^{k}\right) \cap\left(x_{1}^{j}, x_{2}^{j}\right)=\emptyset$,

(iv) for every $a<\infty$, there is only a finite number of $k$ such that $\left(x_{1}^{k}, x_{2}^{k}\right) \cap(-a, a) \neq$ $\emptyset$

(v) for every $k$ there exists $\alpha$ such that $K\left(Q_{k}\right)=\left(x_{1}^{k}, x_{2}^{k}\right) \times\{\alpha\}$,

(vi) for every $k$ there exists $j$ such that

$$
\begin{array}{r}
\int_{\left(\alpha_{1}^{k}, \alpha_{2}^{k}\right)} \sin \alpha d \alpha=\int_{\left(\alpha_{1}^{j}, \alpha_{2}^{j}\right)} \sin \alpha d \alpha, \\
\left(x_{1}^{k}, x_{2}^{k}\right)=\left(x_{1}^{j}, x_{2}^{j}\right), K\left(Q_{k}\right) \subset Q_{j} \text { and } K\left(Q_{j}\right) \subset Q_{k} .
\end{array}
$$

We will call $\sup _{k}\left(x_{2}^{k}-x_{1}^{k}\right) \vee \sup _{k}\left(\alpha_{2}^{k}-\alpha_{1}^{k}\right)$ the mesh of $K$.

Recall from Section 2 that for $K: D \rightarrow D$, the kernel $\mathbb{P}_{K}$ is defined by $\mathbb{P}_{K}(x, \alpha ; d y, d \beta)=\delta_{K(x, \alpha)}(y, \beta) d y d \beta$. Suppose that $\widetilde{\mathbb{P}}$ satisfies (2.4). Then standard arguments (see the proof of Theorem 2.3 in Section 5) show that there exists a sequence $\left\{\widehat{K}_{n}\right\}_{n \geq 1}$ of simple symmetric functions such that $\mathbb{P}_{\widehat{K}_{n}}(x, \alpha ; d y, d \beta) \rightarrow$ $\delta_{x}(y) d y \widetilde{\mathbb{P}}(x, \alpha ; d \beta)$ weakly as $n \rightarrow \infty$. Note that we do not claim that $\widehat{K}_{n}$ arise as functions associated to reflecting sets in $\mathcal{M}_{2}$. To prove Theorem 2.3 , it will suffice to show that for any simple symmetric function $\widehat{K}_{n}: D \rightarrow D$, there exists a sequence of sets $M_{n} \in \mathcal{M}_{2}, n \geq 1$, satisfying conditions (a) and (b) of Theorem 2.3 and such that if the functions $K_{n}: D \rightarrow D$ correspond to $M_{n}$ 's, then $K_{n}-\widehat{K}_{n} \rightarrow 0$ pointwise. The rest of the paper is devoted to the construction of sets $M_{n}$.

For $\rho>0$, let $\Lambda_{\rho}$ be the measure $\Lambda$ restricted to $D_{\rho}:=(-\rho, \rho) \times(-\pi, 0)$ and note that the total mass of $\Lambda_{\rho}$ is $4 \rho$.

Lemma 4.2. For any $\varepsilon_{0}>0$ there exist $n_{0}<\infty$ and $\rho_{0}>0$ such that for any $n>n_{0}, \rho \in\left(0, \rho_{0}\right)$ and any simple symmetric function $K$ with mesh less than or equal to $1 / n$ such that $(-\rho, \rho)$ is one of the intervals $\left(x_{1}^{k}, x_{2}^{k}\right)$, the following holds: $4 \rho \varepsilon_{0}$.

(i) There exist a bounded set $N \in \mathcal{M}_{1}$ and a set $D_{\rho}^{*} \subset D_{\rho}$ such that $\Lambda_{\rho}\left(D_{\rho}^{*}\right)<$

(ii) The function $K_{N}(x, \alpha)$ corresponding to $N$ is defined on $D_{\rho} \backslash D_{\rho}^{*}$.

(iii) For all $(x, \alpha) \in D_{\rho} \backslash D_{\rho}^{*}$, we have $\left|K_{N}(x, \alpha)-K(x, \alpha)\right| \leq \varepsilon_{0}$. 
The function $K_{N}(x, \alpha)$ is not necessarily defined for all $(x, \alpha) \in D_{\rho}$ because some light rays starting from $L_{*}$ and reflected in $N$ may never come back to $L_{*}$.

Proof of Lemma 4.2. We will omit some details of our arguments and estimates that are totally elementary but are tedious to write down. However, we will now provide a solid justification for the approximate formulas that will form the core of the proof. Suppose that two (parts of) ellipses are fixed and serve as mirrors for light rays. Suppose that a light ray starts from $x \in L_{*}$ at an angle $\alpha \in(-\pi, 0)$, then makes two reflections from the elliptic mirrors, and then returns to $L_{*}$ at $y \in L_{*}$ and angle $\beta$. An important (but elementary) observation is that the function $(x, \alpha) \rightarrow(y, \beta)$ is analytic. The reason is that the equations that determine the points and angles of reflection of the light ray are quadratic with "parameters" that are analytic functions of $x$ and $\alpha$. A similar remark applies to other quantities that are functions of $x$ and $\alpha$, such as the location and angle of incidence when the light ray hits one of the ellipses, or the distance from the light ray to a fixed point.

Step 1. In this step, we will construct a pair of elliptic mirrors that interchange two very thin bundles of light rays. This step is devoted only to the construction (or, in other words, definition) of the two mirrors. The next step will contain the proof that the mirrors reflect the light rays in the desired directions.

We will use letters $A, B$ and $C$ (with subscripts and superscripts) to denote points in the plane. The notation $\overline{A B}$ will refer to a line segment with given endpoints; $\overline{A B C}$ will denote an arc of an ellipse passing through the three points.

Consider some (small) $c_{1}>0, \alpha, \beta \in\left(-\pi+c_{1},-c_{1}\right)$ and (small) $\Delta \alpha, \Delta \beta \in(0,1)$, such that $\Delta \alpha \sin \alpha=\Delta \beta \sin \beta$. Suppose that $\rho>0$ and (see Figure 1),

$$
A_{1}=(-\rho, 0), A_{2}=(0,0), A_{3}=(\rho, 0) \text {. }
$$

First assume that $\alpha=\beta$. In this case, let $\mathcal{E}_{\alpha}=\mathcal{E}_{\beta}$ be the arc of a circle represented in complex notation as $\mathcal{E}_{\alpha}=\left\{r e^{i \gamma}: \alpha-\Delta \alpha \leq \gamma \leq \alpha+\Delta \alpha\right\}$ for some $r>0$. Note that the circle is centered at $A_{2}$. We will consider $\mathcal{E}_{\alpha}$ to be a mirror. Light rays starting from $(x, \gamma)$ in $D_{\alpha}^{1}:=\overline{A_{1} A_{3}} \times(\alpha-\Delta \alpha, \alpha+\Delta \alpha)$ will mostly return to the same set if $\rho$ is small. More precisely,

$$
\begin{gathered}
\forall c_{1}>0 \forall \eta_{1}>0 \exists \rho_{0}<\infty \forall \rho \in\left(0, \rho_{0}\right) \forall \alpha \in\left(-\pi+c_{1},-c_{1}\right): \\
\frac{\Lambda_{\rho}\left(\left\{(x, \gamma) \in D_{\alpha}^{1}: K_{\mathcal{E}_{\alpha}}(x, \gamma) \in D_{\alpha}^{1}\right\}\right)}{\Lambda_{\rho}\left(D_{\alpha}^{1}\right)}>1-\eta_{1} .
\end{gathered}
$$

The case when $\alpha=\beta$ is rather easy, so we will focus on the case $\alpha \neq \beta$ in the rest of the proof.

Recall notation from Definition 4.1 and let $\widehat{\alpha}^{k}=\left(\alpha_{1}^{k}+\alpha_{2}^{k}\right) / 2$. Consider $j$ and $k$ such that $K\left(Q_{k}\right) \subset Q_{j}$ and $K\left(Q_{j}\right) \subset Q_{k}$. If $\left(\alpha_{2}^{k}-\alpha_{1}^{j}\right) \vee\left(\alpha_{2}^{j}-\alpha_{1}^{k}\right) \leq \varepsilon_{0} / 2$, then let $K^{\prime}(x, \alpha)=\left(x, \widehat{\alpha}^{k}\right)$ for $(x, \alpha) \in Q_{k}$, and $K^{\prime}(x, \alpha)=\left(x, \widehat{\alpha}^{j}\right)$ for $(x, \alpha) \in Q_{j}$. Otherwise, we let $K^{\prime}(x, \alpha)=K(x, \alpha)$. Note that $K^{\prime}$ is a simple symmetric function and $\left|K^{\prime}(x, \alpha)-K(x, \alpha)\right| \leq \varepsilon_{0} / 2$. Hence, it will suffice to find a set $N$ and the corresponding function $K_{N}$ such that $\left|K_{N}(x, \alpha)-K^{\prime}(x, \alpha)\right| \leq \varepsilon_{0} / 2$.

Consider the case when $|\alpha-\beta| \geq \varepsilon_{0} / 2$ and $\alpha-\Delta \alpha, \alpha+\Delta \alpha, \beta-\Delta \beta, \beta+\Delta \beta \in$ $(-\pi, 0)$. For any angle $\gamma \in(-\pi, 0)$, let $L_{\gamma}$ be the line passing through $A_{2}$, with the slope $\tan (-\gamma)$. Unless stated otherwise, we will consider only points and sets below the line $L_{*}$. 


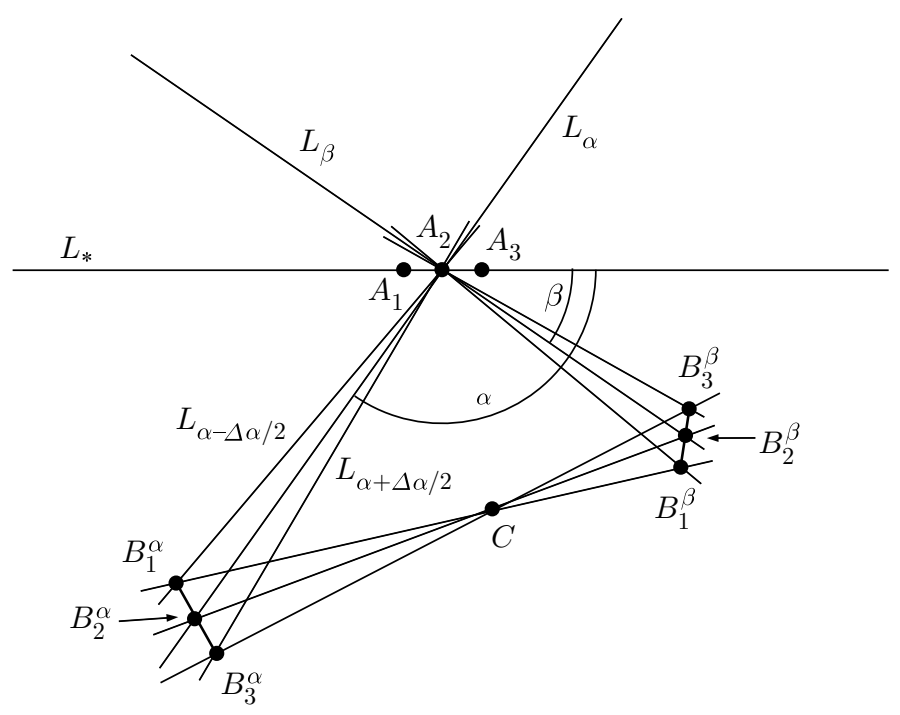

FiguRE 1. A single light ray interchange mirror system.

Let $B_{2}^{\alpha}$ be a point on $L_{\alpha}$, and $r_{\alpha}:=\left|A_{2}-B_{2}^{\alpha}\right|$. Similarly, let $B_{2}^{\beta}$ be a point on $L_{\beta}$, and $r_{\beta}:=\left|A_{2}-B_{2}^{\beta}\right|$ (values of $r_{\alpha}$ and $r_{\beta}$ will be specified later). Let $L^{\prime}$ be the line passing through $B_{2}^{\alpha}$ and $B_{2}^{\beta}$, and let $C$ be the point on $L^{\prime}$ such that $s_{1}:=\left|C-B_{2}^{\alpha}\right|=\left|C-B_{2}^{\beta}\right|$.

Recall that for any ellipse, if a light ray leaves one of the foci and meets a point on that ellipse, it will reflect off the ellipse and pass through the other focus.

Let $B_{1}^{\alpha} \in L_{\alpha-\Delta \alpha / 2}$ and $B_{3}^{\alpha} \in L_{\alpha+\Delta \alpha / 2}$ be points such that $\mathcal{E}_{\alpha}:=\overline{B_{1}^{\alpha} B_{2}^{\alpha} B_{3}^{\alpha}}$ is an arc of an ellipse with the foci $A_{2}$ and $C$ (see Figure1). Similarly, let $B_{1}^{\beta} \in L_{\beta-\Delta \beta / 2}$ and $B_{3}^{\beta} \in L_{\beta+\Delta \beta / 2}$ be points such that $\mathcal{E}_{\beta}:=\overline{B_{1}^{\beta} B_{2}^{\beta} B_{3}^{\beta}}$ is an arc of an ellipse with the foci $A_{2}$ and $C$.

The rest of this step is devoted to informal estimates aimed at finding values of $r_{\alpha}$ and $r_{\beta}$ that will define mirrors with desirable properties. Consider the bundle $\mathcal{B}$ of light rays starting at $A_{2}$ along lines between $L_{\alpha-\Delta \alpha / 2}$ and $L_{\alpha+\Delta \alpha / 2}$. We will find parameters of our construction such that for the resulting mirrors $\mathcal{E}_{\alpha}$ and $\mathcal{E}_{\beta}$, "most" light rays in $\mathcal{B}$ reflect from $\mathcal{E}_{\alpha}$, then reflect from $\mathcal{E}_{\beta}$ and then hit $A_{2}$. Let $\rho_{1}$ be the width of $\mathcal{B}$ close to $\mathcal{E}_{\alpha}$, just before hitting $\mathcal{E}_{\alpha}$. Since $\Delta \alpha$ is assumed to be small, the rays in $\mathcal{B}$ are almost parallel and $\mathcal{E}_{\alpha}$ is almost flat. Hence, the width of $\mathcal{B}$ close to $\mathcal{E}_{\alpha}$, just after hitting $\mathcal{E}_{\alpha}$ is $\rho_{1}(1+o(\Delta \alpha))$. We have $\rho_{1}=r_{\alpha}(\Delta \alpha+o(\Delta \alpha))$ for small $\Delta \alpha$.

Since $\mathcal{E}_{\alpha}$ is an arc of an ellipse with foci $A_{2}$ and $C$, all light rays in $\mathcal{B}$ will pass through $C$ and then they will hit $\mathcal{E}_{\beta}$, assuming that $\mathcal{E}_{\beta}$ is large enough. Since $\mathcal{E}_{\beta}$ is an arc of an ellipse with foci $A_{2}$ and $C$, the light rays in $\mathcal{B}$ reflected from $\mathcal{E}_{\beta}$ will hit $L_{*}$ at $A_{2}$.

Let $\rho_{2}$ be the width of $\mathcal{B}$ close to $\mathcal{E}_{\beta}$, just before hitting $\mathcal{E}_{\beta}$. The width of $\mathcal{B}$ close to $\mathcal{E}_{\beta}$, just after hitting $\mathcal{E}_{\beta}$, is $\rho_{2}(1+o(\Delta \alpha))$. We want the bundle of light rays $\mathcal{B}$ to form a cone with angle $\Delta \beta$ at the point $A_{2}$ on the way out, so we would like to have $\rho_{2}=r_{\beta}(\Delta \beta+o(\Delta \beta))$ for small $\Delta \beta$. The point $C$ is half way between $B_{2}^{\alpha}$ and 
$B_{2}^{\beta}$, so we would like to have $\rho_{1}=\rho_{2}$. Therefore, we choose $r_{\alpha}$ and $r_{\beta}$ so that they satisfy

$$
r_{\alpha}(\Delta \alpha+o(\Delta \alpha))=\rho_{1}=\rho_{2}=r_{\beta}(\Delta \beta+o(\Delta \beta)) .
$$

More precisely, we choose $r_{\alpha}$ and $r_{\beta}$ so that

$$
\frac{r_{\alpha}}{r_{\beta}}=\frac{\Delta \beta}{\Delta \alpha}=\frac{\sin \alpha}{\sin \beta}
$$

The above formula incorporates our previously made assumption that $\Delta \alpha \sin \alpha=$ $\Delta \beta \sin \beta$.

Step 2. We will now argue that a pair of mirrors defined in the previous step guides a thin bundle of light rays to the appropriate exit location and exit angle, with arbitrarily little "waste", for appropriate values of parameters of the construction. Our argument is based on detailed analysis of small changes in the initial conditions (the starting location and angle of the light ray) on the "output", that is, the location and angle for the light ray exiting the lower half-plane.

Let $\mathbf{E}_{\alpha}$ be the ellipse of which $\mathcal{E}_{\alpha}$ is a part and let $\mathbf{E}_{\beta}$ have the analogous meaning. The informal estimates of Step 1 have the following rigorous version. For any $c_{1}, \varepsilon>$ 0 , there exists $\varepsilon_{1} \in(0, \varepsilon)$ such that for all $\alpha, \beta \in\left(-\pi+c_{1},-c_{1}\right)$ with $|\alpha-\beta| \geq \varepsilon_{0} / 2$, $\Delta \alpha, \Delta \beta \in\left(0, \varepsilon_{1}\right)$, and $r_{\alpha}$ and $r_{\beta}$ satisfying (4.1), we have the following. If a light ray starts from $A_{2}$, follows $L_{\alpha+\alpha_{1}}$ with $\alpha_{1} \in\left[-\left(1-\varepsilon_{1}\right) \Delta \alpha / 2,\left(1-\varepsilon_{1}\right) \Delta \alpha / 2\right]$ and reflects from $\mathcal{E}_{\alpha}$, then it will intersect $\mathbf{E}_{\beta}$ at a point $x$ such that

$$
\left|x-B_{2}^{\beta}\right|<(1-\varepsilon)\left(\left|B_{1}^{\beta}-B_{2}^{\beta}\right| \vee\left|B_{3}^{\beta}-B_{2}^{\beta}\right|\right) .
$$

Similarly, if a light ray starts from $A_{2}$, follows $L_{\beta+\beta_{1}}$ with $\beta_{1} \in\left[-\left(1-\varepsilon_{1}\right) \Delta \beta / 2,(1-\right.$ $\left.\varepsilon_{1}\right) \Delta \beta / 2$ ] and reflects from $\mathcal{E}_{\beta}$, then it will intersect $\mathbf{E}_{\alpha}$ at a point $y$ such that

$$
\left|y-B_{2}^{\alpha}\right|<(1-\varepsilon)\left(\left|B_{1}^{\alpha}-B_{2}^{\alpha}\right| \vee\left|B_{3}^{\alpha}-B_{2}^{\alpha}\right|\right) .
$$

It follows from (4.2) and (4.3) that all light rays starting from $A_{2}$ at angles in $\left[-\left(1-\varepsilon_{1}\right) \Delta \alpha / 2,\left(1-\varepsilon_{1}\right) \Delta \alpha / 2\right]$ or $\left[-\left(1-\varepsilon_{1}\right) \Delta \beta / 2,\left(1-\varepsilon_{1}\right) \Delta \beta / 2\right]$ reflect from both mirrors $\mathcal{E}_{\alpha}$ and $\mathcal{E}_{\beta}$ and then reach $L_{*}$ again at $A_{2}$.

In the following part of the argument, we will consider $\alpha, \beta, r_{\alpha}$ and $r_{\beta}$ to be "fixed" parameters satisfying (4.1) and we will choose $\Delta \alpha, \Delta \beta$ and $\rho$ sufficiently small, so that certain conditions are satisfied.

We will define a number of arcs, lines and points which will be used in the next part of the proof; see Figure 2. Consider any point $A_{4} \in \overline{A_{1} A_{3}}$ and light ray $R_{1}$ emanating from $A_{4}$ at an angle $\eta$. Let $B_{4} \in \mathbf{E}_{\alpha}$ and $B_{6} \in \mathbf{E}_{\beta}$ denote the points where this light ray reflects in $\mathbf{E}_{\alpha}$ and $\mathbf{E}_{\beta}$. Let $R_{4}$ be the line between $B_{4}$ and $B_{6}$. Let $R_{6}$ be the line that contains this light ray after reflection in $\mathbf{E}_{\beta}$ and let $B_{8}$ be the point where $R_{6}$ intersects $L_{*}$. Let $R_{2}$ be the line passing through $A_{2}$ and parallel to $R_{1}$ and let $B_{5}$ be the intersection point of $R_{2}$ with $\mathbf{E}_{\alpha}$. Let $R_{3}$ be the line passing through $A_{2}$ and $B_{4}$. Let $R_{5}$ be the line that represents light ray $R_{3}$ after reflecting from $\mathbf{E}_{\alpha}$. Let $B_{7}$ be the point where $R_{5}$ intersects $\mathbf{E}_{\beta}$. Let $R_{7}$ be the line passing through $A_{2}$ and $B_{6}$.

Let $\alpha_{1}$ be the angle between $\overline{A_{2} B_{2}^{\alpha}}$ and the tangent to $\mathcal{E}_{\alpha}$ at $B_{2}^{\alpha}$. The angle $\alpha_{1}$ is a function of $\alpha, \beta, r_{\alpha}$ and $r_{\beta}$ only. Assume that $\eta \in\left[\alpha-\left(1-\varepsilon_{1}\right) \Delta \alpha / 2\right.$, 


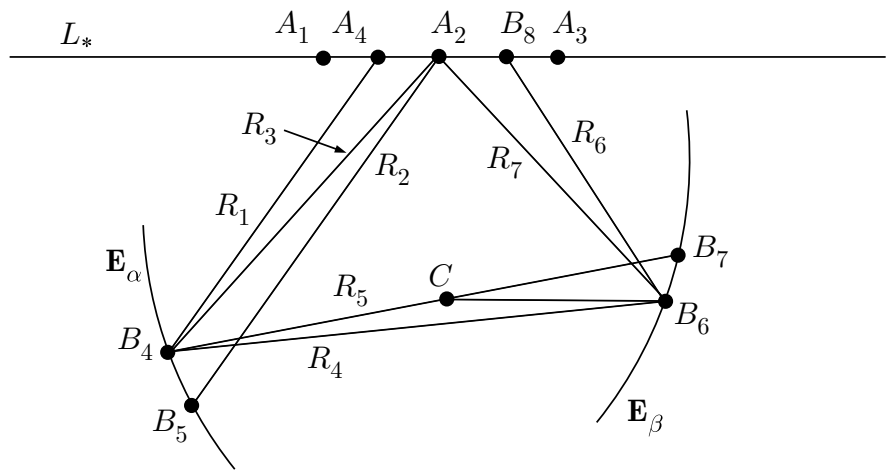

FiguRE 2. Trajectories of various light rays.

$\left.\alpha+\left(1-\varepsilon_{1}\right) \Delta \alpha / 2\right]$. Then

$$
\begin{aligned}
\left|B_{5}-B_{2}^{\alpha}\right| & \leq\left(1-\varepsilon_{1}+o\left(\varepsilon_{1}\right)\right)\left(\left|B_{1}^{\alpha}-B_{2}^{\alpha}\right| \vee\left|B_{3}^{\alpha}-B_{2}^{\alpha}\right|\right) \\
& \leq\left(1-\varepsilon_{1}+o\left(\varepsilon_{1}\right)\right) \frac{r_{\alpha}(\Delta \alpha / 2)(1+o(\Delta \alpha))}{\sin \alpha_{1}}
\end{aligned}
$$

We also have

$$
\left|B_{4}-B_{5}\right| \leq(1+o(\rho)) \frac{\rho \sin \alpha}{\sin \alpha_{1}}
$$

and, therefore,

$$
\left|B_{4}-B_{2}^{\alpha}\right| \leq\left(1-\varepsilon_{1}+o\left(\varepsilon_{1}\right)\right) \frac{r_{\alpha}(\Delta \alpha / 2)(1+o(\Delta \alpha))}{\sin \alpha_{1}}+(1+o(\rho)) \frac{\rho \sin \alpha}{\sin \alpha_{1}} .
$$

It follows that for any $c_{1}, \varepsilon>0$, there exists $\varepsilon_{1} \in(0, \varepsilon), \varepsilon_{2}>0$, such that for all $\alpha, \beta \in\left(-\pi+c_{1},-c_{1}\right)$ with $|\alpha-\beta| \geq \varepsilon_{0} / 2$ and $\Delta \alpha, \Delta \beta \in\left(0, \varepsilon_{1}\right), r_{\alpha}$ and $r_{\beta}$ satisfying (4.1), all

$$
\rho \in\left(0, \varepsilon_{2}(\Delta \alpha \wedge \Delta \beta)\right) \text { and } \eta \in\left[\alpha-\left(1-\varepsilon_{1}\right) \Delta \alpha / 2, \alpha+\left(1-\varepsilon_{1}\right) \Delta \alpha / 2\right],
$$

we have $B_{4} \in \mathcal{E}_{\alpha}$. By symmetry, if $\eta \in\left[\beta-\left(1-\varepsilon_{1}\right) \Delta \beta / 2, \beta+\left(1-\varepsilon_{1}\right) \Delta \beta / 2\right]$, then $R_{1}$ intersects $\mathbf{E}_{\beta}$ at a point in $\mathcal{E}_{\beta}$.

Suppose that $\eta \in\left[\alpha-\left(1-\varepsilon_{1}\right) \Delta \alpha / 2, \alpha+\left(1-\varepsilon_{1}\right) \Delta \alpha / 2\right]$. Let $\beta_{1}$ be the angle between $\overline{A_{2} B_{2}^{\beta}}$ and the tangent to $\mathcal{E}_{\beta}$ at $B_{2}^{\beta}$. Note that $\beta_{1}$ is also the angle between $\overline{C B_{2}^{\beta}}$ and the tangent to $\mathcal{E}_{\beta}$ at $B_{2}^{\beta}$. Let $\gamma_{1}$ be the angle between $R_{1}$ and $R_{3}$. The angle between $R_{4}$ and $R_{5}$ is also $\gamma_{1}$. We have

$$
\gamma_{1}=\sin \alpha\left|A_{4}-A_{2}\right|(1+o(\rho)+o(\Delta \alpha)) / r_{\alpha} \leq \rho \sin \alpha(1+o(\rho)+o(\Delta \alpha)) / r_{\alpha} .
$$

Thus

$$
\begin{aligned}
\left|B_{6}-B_{7}\right| & =\frac{2 s_{1} \sin \gamma_{1}}{\sin \beta_{1}}(1+o(\rho)+o(\Delta \beta)) \\
& \leq \frac{2 s_{1} \rho \sin \alpha(1+o(\rho)+o(\Delta \alpha))}{r_{\alpha} \sin \beta_{1}}(1+o(\rho)+o(\Delta \beta)) \\
& =\frac{2 s_{1} \rho \sin \alpha}{r_{\alpha} \sin \beta_{1}}(1+o(\rho)+o(\Delta \beta)) .
\end{aligned}
$$


It follows from (4.2), (4.3) and (4.4) that for any $c_{1}, \varepsilon>0$ there exists $\varepsilon_{1} \in(0, \varepsilon)$, $\varepsilon_{2}>0$ such that for all $\alpha, \beta \in\left(-\pi+c_{1},-c_{1}\right)$ with $|\alpha-\beta| \geq \varepsilon_{0} / 2$ and $\Delta \alpha, \Delta \beta \in$ $\left(0, \varepsilon_{1}\right), r_{\alpha}$ and $r_{\beta}$ satisfying (4.1), and all $\rho \in\left(0, \varepsilon_{2}(\Delta \alpha \wedge \Delta \beta)\right)$ we have the following. If a light ray starts from a point in $\overline{A_{1} A_{3}}$ at an angle $\eta \in\left[\alpha-\left(1-\varepsilon_{1}\right) \Delta \alpha / 2, \alpha+(1-\right.$ $\left.\varepsilon_{1}\right) \Delta \alpha / 2$, then it reflects from $\mathcal{E}_{\alpha}$ and then it intersects $\mathcal{E}_{\beta}$. Similarly, if a light ray starts from a point in $\overline{A_{1} A_{3}}$ at an angle $\eta \in\left[\beta-\left(1-\varepsilon_{1}\right) \Delta \beta / 2, \beta+\left(1-\varepsilon_{1}\right) \Delta \beta / 2\right]$, then it reflects from $\mathcal{E}_{\beta}$ and then it intersects $\mathcal{E}_{\alpha}$.

Let $a$ denote the distance between $C$ and $R_{4}$. We have

$$
a=s_{1} \sin \gamma_{1}(1+o(\rho))=s_{1} \sin \alpha\left|A_{4}-A_{2}\right|(1+o(\rho)+o(\Delta \alpha)) / r_{\alpha} .
$$

Note that if a light ray moved along $\overline{C B_{6}}$ and reflected from $\mathbf{E}_{\beta}$, then it stays on $R_{7}$. The angle between $R_{6}$ and $R_{7}$ is equal to the angle between $\overline{C B_{6}}$ and $R_{4}$ and, therefore, it is equal to $\gamma_{2}:=\left(a / s_{1}\right)(1+o(\rho)+o(\Delta \alpha))$. The following estimate for the distance between $A_{2}$ and $B_{8}$ uses (4.1),

$$
\begin{aligned}
\left|B_{8}-A_{2}\right| & =\frac{\gamma_{2} r_{\beta}}{\sin \beta}(1+o(\Delta \alpha))=\frac{a r_{\beta}}{s_{1} \sin \beta}(1+o(\rho)+o(\Delta \alpha)) \\
& =\frac{s_{1} \sin \alpha\left|A_{4}-A_{2}\right| r_{\beta}}{r_{\alpha} s_{1} \sin \beta}(1+o(\rho)+o(\Delta \alpha)) \\
& =\left|A_{4}-A_{2}\right|(1+o(\rho)+o(\Delta \alpha)) .
\end{aligned}
$$

We combine this estimate with the conclusions obtained so far to see that for any $c_{1}, \varepsilon>0$ there exists $\varepsilon_{1} \in(0, \varepsilon), \varepsilon_{2}>0$ such that for all $\alpha, \beta \in\left(-\pi+c_{1},-c_{1}\right)$ with $|\alpha-\beta| \geq \varepsilon_{0} / 2$ and $\Delta \alpha, \Delta \beta \in\left(0, \varepsilon_{1}\right), r_{\alpha}$ and $r_{\beta}$ satisfying (4.1), all $\rho \in$ $\left(0, \varepsilon_{2}(\Delta \alpha \wedge \Delta \beta)\right)$, and all $A_{4} \in \overline{A_{1} A_{3}}$ satisfying $\left|A_{4}-A_{2}\right| \leq\left(1-\varepsilon_{2}\right) \rho$ we have the following. If a light ray starts from $A_{4}$ at an angle $\eta \in\left[\alpha-\left(1-\varepsilon_{1}\right) \Delta \alpha / 2, \alpha+(1-\right.$ $\left.\varepsilon_{1}\right) \Delta \alpha / 2$, then it reflects from $\mathcal{E}_{\alpha}$, next it reflects from $\mathcal{E}_{\beta}$ and then it intersects $L_{*}$ between $A_{1}$ and $A_{3}$. Similarly, if a light ray starts from $A_{4}$ at an angle $\eta \in$ $\left[\beta-\left(1-\varepsilon_{1}\right) \Delta \beta / 2, \beta+\left(1-\varepsilon_{1}\right) \Delta \beta / 2\right]$, then it reflects from $\mathcal{E}_{\beta}$, next it reflects from $\mathcal{E}_{\alpha}$ and then it intersects $L_{*}$ between $A_{1}$ and $A_{3}$.

Step 3. We will now assemble a finite family of pairs of mirrors so that they properly guide light rays entering the system at "most" angles and the mirrors do not interfere with one another. The lack of interference will be achieved by inductive scaling of the mirrors, that is, making them large, so that a light ray traveling between a pair of mirrors takes a path far beyond all the mirrors constructed earlier in the inductive procedure.

Recall the notation from the statement of the lemma. Let $c_{1}>0$ be so small that

$$
\Lambda_{\rho}\left(D_{\rho} \backslash\left((-\rho, \rho) \times\left(-\pi+c_{1},-c_{1}\right)\right)\right)<4 \rho \varepsilon_{0} / 16
$$

Note that $c_{1}$ satisfying this condition can be chosen independently of $\rho$.

Recall the simple symmetric function $K^{\prime}$ defined in Step 1 and let $\left\{Q_{k}^{\prime}\right\}_{k \geq 1}=$ $\left\{\left(x_{1}^{k}, x_{2}^{k}\right) \times\left(\alpha_{1}^{k}, \alpha_{2}^{k}\right)\right\}_{k \geq 1}$ be the family of rectangles as in Definition 4.1. Let $\mathbf{J}$ be the family of pairs $(j, k)$ such that $j \leq k$,

$$
K^{\prime}\left((-\rho, \rho) \times\left(\alpha_{1}^{j}, \alpha_{2}^{j}\right)\right) \subset(-\rho, \rho) \times\left(\alpha_{1}^{k}, \alpha_{2}^{k}\right)
$$

and, therefore,

$$
K^{\prime}\left((-\rho, \rho) \times\left(\alpha_{1}^{k}, \alpha_{2}^{k}\right)\right) \subset(-\rho, \rho) \times\left(\alpha_{1}^{j}, \alpha_{2}^{j}\right)
$$


Note that according to Definition 4.1)(iv), the set $\mathbf{J}$ is finite. Let $\mathcal{J}$ be the set of all $j$ such that $\left[\alpha_{1}^{j}, \alpha_{2}^{j}\right] \cap\left(-\pi+c_{1},-c_{1}\right) \neq \emptyset$. For $(j, k) \in \mathbf{J}$, let

$$
\begin{aligned}
\widehat{\alpha}^{j} & =\left(\alpha_{1}^{j}+\alpha_{2}^{j}\right) / 2, \\
\Delta \widehat{\alpha}^{j} & =\alpha_{2}^{j}-\alpha_{1}^{j}, \\
\Delta \widehat{\alpha}^{k} & =\Delta \widehat{\alpha}^{j} \sin \widehat{\alpha}^{j} / \sin \widehat{\alpha}^{k} .
\end{aligned}
$$

Note that, typically, it is not true that $\Delta \widehat{\alpha}^{k}=\alpha_{2}^{k}-\alpha_{1}^{k}$ because if $(j, k) \in \mathbf{J}$ and $j \neq k$, then $(k, j) \notin \mathbf{J}$. The quantity $\Delta \widehat{\alpha}^{k}$ is well defined because if $(j, k) \in \mathbf{J}$ and $j \neq k$, then there is no $i$ such that $(k, i) \in \mathbf{J}$. It is easy to see that for fixed $c_{1}, \varepsilon_{1}>0$, we can choose $n_{0}$ so large that for all $n \geq n_{0}$, if the mesh of $K$ is smaller than $1 / n$, then for all $j \in \mathcal{J}$ we have

$$
\left[\widehat{\alpha}^{j}-\left(1-\varepsilon_{1}\right) \Delta \widehat{\alpha}^{j} / 2, \widehat{\alpha}^{j}+\left(1-\varepsilon_{1}\right) \Delta \widehat{\alpha}^{j} / 2\right] \subset\left(\alpha_{1}^{j}, \alpha_{2}^{j}\right) .
$$

Let

$$
\Theta\left(\varepsilon_{1}\right)=\left(-\pi+c_{1},-c_{1}\right) \cap \bigcup_{j \in \mathcal{J}}\left[\widehat{\alpha}^{j}-\left(1-\varepsilon_{1}\right) \Delta \widehat{\alpha}^{j} / 2, \widehat{\alpha}^{j}+\left(1-\varepsilon_{1}\right) \Delta \widehat{\alpha}^{j} / 2\right] .
$$

For a fixed $c_{1}>0$, we can make $\varepsilon_{1}>0$ smaller and $n_{0}$ larger, if necessary, so that

$$
\Lambda_{\rho}\left((-\rho, \rho) \times \Theta\left(\varepsilon_{1}\right)\right)>4 \rho\left(1-\varepsilon_{0} / 8\right) .
$$

We make $n_{0}$ larger, if necessary, so that for $n \geq n_{0}$ and all $j \in \mathcal{J}$ we have $\Delta \widehat{\alpha}^{j}<\varepsilon_{1}$.

Choose $\varepsilon_{3}>0$ so small that

$$
\Lambda_{\rho}\left(\left(-\left(1-\varepsilon_{3}\right) \rho,\left(1-\varepsilon_{3}\right) \rho\right) \times \Theta\left(\varepsilon_{1}\right)\right)>4 \rho\left(1-\varepsilon_{0} / 4\right) .
$$

We will write $r(\alpha)$ instead of $r_{\alpha}$ and $\mathcal{E}(\alpha)$ instead of $\mathcal{E}_{\alpha}$, for typographical reasons.

Let $\Delta^{*} \alpha=\min _{j \in \mathcal{J}} \Delta \widehat{\alpha}^{j}$. It follows from what we have shown in Step 2 that for $c_{1}, \varepsilon>0$ there exists $n_{0}, \varepsilon_{1} \in(0, \varepsilon), \varepsilon_{2}>0$ such that for all $\rho \in\left(0, \varepsilon_{2} \Delta^{*} \alpha\right), n \geq n_{0}$ and all $j \in \mathcal{J}$ we have the following. If $(j, k) \in \mathbf{J}$ and a light ray starts from a point in $\left(-\left(1-\varepsilon_{3}\right) \rho,\left(1-\varepsilon_{3}\right) \rho\right) \subset L_{*}$ at an angle

$$
\eta \in\left[\widehat{\alpha}^{j}-\left(1-\varepsilon_{1}\right) \Delta \widehat{\alpha}^{j} / 2, \widehat{\alpha}^{j}+\left(1-\varepsilon_{1}\right) \Delta \widehat{\alpha}^{j} / 2\right],
$$

then it reflects from $\mathcal{E}\left(\widehat{\alpha}^{j}\right)$, next it reflects from $\mathcal{E}\left(\widehat{\alpha}^{k}\right)$ and then it intersects $L_{*}$ at a point in $\left(-\left(1-\varepsilon_{3}\right) \rho,\left(1-\varepsilon_{3}\right) \rho\right)$. Moreover, if a light ray starts from a point in $\left(-\left(1-\varepsilon_{3}\right) \rho,\left(1-\varepsilon_{3}\right) \rho\right) \subset L_{*}$ at an angle

$$
\eta \in\left[\widehat{\alpha}^{k}-\left(1-\varepsilon_{1}\right) \Delta \widehat{\alpha}^{k} / 2, \widehat{\alpha}^{k}+\left(1-\varepsilon_{1}\right) \Delta \widehat{\alpha}^{k} / 2\right],
$$

then it reflects from $\mathcal{E}\left(\widehat{\alpha}^{k}\right)$, next it reflects from $\mathcal{E}\left(\widehat{\alpha}^{j}\right)$ and then it intersects $L_{*}$ at a point in $\left(-\left(1-\varepsilon_{3}\right) \rho,\left(1-\varepsilon_{3}\right) \rho\right)$.

Let $\left(j_{1}, k_{1}\right),\left(j_{2}, k_{2}\right), \ldots$ be an arbitrary ordering of pairs in $\mathbf{J}$. Choose $r\left(\widehat{\alpha}^{j_{1}}\right)$ and $r\left(\widehat{\alpha}^{k_{1}}\right)$ so that they satisfy (4.1). Suppose that $r\left(\widehat{\alpha}^{j_{m}}\right)$ and $r\left(\widehat{\alpha}^{k_{m}}\right)$ have been chosen for $m=1,2, \ldots, i$. We choose $r\left(\widehat{\alpha}^{j_{i+1}}\right)$ and $r\left(\widehat{\alpha}^{k_{i+1}}\right)$ so that they satisfy (4.1) and they are so large that lines connecting any points in $\mathcal{E}\left(\widehat{\alpha}^{j_{i+1}}\right)$ and $\mathcal{E}\left(\widehat{\alpha}^{k_{i+1}}\right)$ do not intersect any $\mathcal{E}\left(\widehat{\alpha}^{j_{m}}\right)$ and $\mathcal{E}\left(\widehat{\alpha}^{k_{m}}\right)$ for $m=1,2, \ldots, i$.

Recall that $\mathbf{J}$ is finite and let $n_{1}$ be its cardinality. For $(j, k) \in \mathbf{J}$, let $\widehat{D}_{\rho,(j, k)}$ be the set of light rays in $D_{\rho}$ such that the ray starts from a point in $\left[-\left(1-\varepsilon_{3}\right) \rho,(1-\right.$ $\left.\left.\varepsilon_{3}\right) \rho\right] \subset L_{*}$ at an angle

$$
\eta \in\left[\widehat{\alpha}^{j}-\left(1-\varepsilon_{1}\right) \Delta \widehat{\alpha}^{j} / 2, \widehat{\alpha}^{j}+\left(1-\varepsilon_{1}\right) \Delta \widehat{\alpha}^{j} / 2\right]
$$


or at an angle

$$
\eta \in\left[\widehat{\alpha}^{k}-\left(1-\varepsilon_{1}\right) \Delta \widehat{\alpha}^{k} / 2, \widehat{\alpha}^{k}+\left(1-\varepsilon_{1}\right) \Delta \widehat{\alpha}^{k} / 2\right] .
$$

Let $\widetilde{D}_{\rho,(j, k)}$ be the set of light rays in $\widehat{D}_{\rho,(j, k)}$ such that at some time the ray reflects from a set $\mathcal{E}\left(\widehat{\alpha}^{m}\right)$, for some $m \neq j, k$. If $\rho=0$, that is, if $\left[-\left(1-\varepsilon_{3}\right) \rho,\left(1-\varepsilon_{3}\right) \rho\right]$ is the single point $A_{2}$, then $\widetilde{D}_{\rho,(j, k)}=\emptyset$, by the claim made in the previous paragraph. The proportion (in terms of the measure $\Lambda_{\rho}$ ) of light rays in $\widehat{D}_{\rho,(j, k)}$ which belong to $\widetilde{D}_{\rho,(j, k)}$ is a continuous function of $\rho>0$, with zero limit when $\rho \downarrow 0$; that is, $\lim _{\rho \downarrow 0} \Lambda_{\rho}\left(\widetilde{D}_{\rho,(j, k)}\right) / \Lambda_{\rho}\left(\widehat{D}_{\rho,(j, k)}\right)=0$. It follows that for sufficiently small $\rho>0$, we have $\Lambda_{\rho}\left(\widetilde{D}_{\rho,(j, k)}\right)<4 \rho \varepsilon_{0} /\left(16 n_{1}\right)$. Let $\widetilde{D}_{\rho}=\bigcup_{(j, k) \in \mathbf{J}} \widetilde{D}_{\rho,(j, k)}$. We see that for fixed $r\left(\widehat{\alpha}^{j}\right)$ 's, we can make $\rho>0$ so small that $\Lambda_{\rho}\left(\widetilde{D}_{\rho}\right)<4 \rho \varepsilon_{0} / 16$.

Let $N=\bigcup_{j \in \mathcal{J}} \mathcal{E}\left(\widehat{\alpha}^{j}\right)$. Our arguments have shown that the function $K_{N}$ satisfies the assertions listed in the lemma, with function $K^{\prime}$ in place of $K$, and $\varepsilon_{0} / 2$ in place of $\varepsilon_{0}$. We have observed in Step 1 that this is sufficient for the proof of the lemma.

\section{Light Reflector Cells in Cantor Set holes}

The system of mirrors constructed in the previous section is very "inefficient" in that it has a large diameter compared to the length of the segment $(-\rho, \rho)$ of $L_{*}$, where the light enters and exits the system. In this section, we will build a "compact" version of the reflector using scaling and a Cantor-like construction. The following result is very similar to Lemma 4.2 except that we use smaller mirrors.

Lemma 5.1. Fix any $\rho, \rho_{1}>0, a \in \mathbb{R}$ and let $\Lambda_{\rho}$ be the measure $\Lambda$ restricted to $D_{\rho}:=(a-\rho, a+\rho) \times(-\pi, 0)$. The total mass of $\Lambda_{\rho}$ is $4 \rho$. For any $\varepsilon_{0}>0$, there exists $n_{0}<\infty$ such that for any $n>n_{0}$ and any simple symmetric function $K$ with parameter $n$ such that $[a-\rho, a+\rho]$ is one of the intervals $\left[x_{1}^{k}, x_{2}^{k}\right]$, the following holds:

(i) There exist a compact set $N \in \mathcal{M}_{1}$ and a set $D_{\rho}^{*} \subset D_{\rho}$ such that $\Lambda_{\rho}\left(D_{\rho}^{*}\right)<$ $4 \rho \varepsilon_{0}$.

(ii) The function $K_{N}(x, \alpha)$ corresponding to $N$ is defined on $D_{\rho} \backslash D_{\rho}^{*}$.

(iii) For all $(x, \alpha) \in D_{\rho} \backslash D_{\rho}^{*}$, we have $\left|K_{N}(x, \alpha)-K(x, \alpha)\right| \leq \varepsilon_{0}$.

(iv) $N \subset(a-\rho, a+\rho) \times\left(-\rho_{1}, 0\right)$.

Proof. By translation invariance, it is enough to discuss the case $a=0$.

Recall that we consider $(-\rho, \rho)$ to be a subset of the horizontal axis $L_{*}$. Let $N_{0}$ be a new name for the set satisfying Lemma 4.2 and let $M$ be the closure of the convex hull of $N_{0} \cup(-\rho, \rho)$. Since $c_{1}$ in the proof of Lemma 4.2 is strictly positive, $M \cap L_{*}=[-\rho, \rho]$. Let $r_{0}$ be the diameter of $M$ and let $M_{r}=\left\{x \in \mathbb{R}^{2}: \exists y \in\right.$ $M$ such that $x=\left(r / r_{0}\right) y$, i.e., $M_{r}$ is a dilation of $M$.

We will define sets $M_{r}^{j}$. Each of these sets will be a horizontal shift of $M_{r}$; that is, $M_{r}^{j}=M_{r}+(b, 0)$ for some $b \in \mathbb{R}$ depending on $j$ and $r$. For any such set, we let $\widetilde{M}_{r}^{j}=M_{r}^{j} \cap L_{*}$. Note that the length of $\widetilde{M}_{r}^{j}$ is $2\left(r / r_{0}\right) \rho$.

The sets $M_{r}^{j}$ will be grouped into a countable number of families, with all sets in one family having the same size. We will pack smaller sets among the bigger sets in a tight way, as much as possible. The intersections of the sets $M_{r}^{j}$ with $L_{*}$ will form a pattern qualitatively similar to holes in the Cantor set; see Figure 3. 


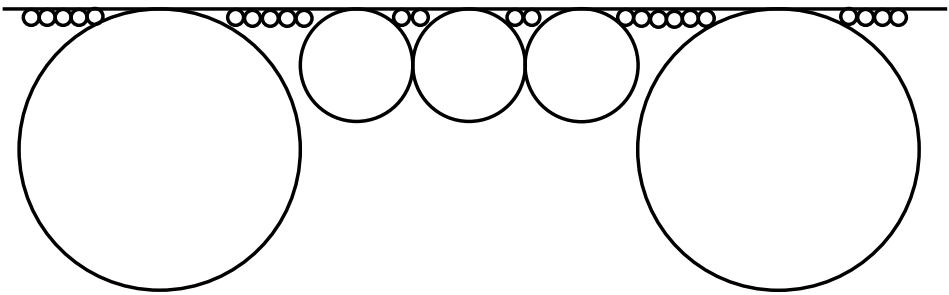

Figure 3. Sets $M_{r_{k}}^{j}$ of three "generations". The sets $M_{r}^{j}$ are convex by definition. They are pictured as circles but in fact they are not discs.

For $r \in(0,1)$, let $n_{r}$ be the maximum number $k$ of disjoint sets $M_{r}^{1}, M_{r}^{2}, \ldots, M_{r}^{k}$ such that $\bigcup_{1 \leq j \leq k} M_{r}^{j} \subset\left\{\left(x_{1}, x_{2}\right):\left|x_{1}\right|<1\right\}$. It is easy to see that for some $c_{2}, r_{*}>0$ and all $r \in\left(0, r_{*}\right)$ we have $r n_{r}>c_{2}$. It follows that for some $c_{3}>0$, for every $r \in\left(0, r_{*}\right)$ one can find a family of disjoint sets $M_{r}^{1}, M_{r}^{2}, \ldots, M_{r}^{k}$ such that $\bigcup_{1 \leq j \leq k} M_{r}^{j} \subset\left\{\left(x_{1}, x_{2}\right):\left|x_{1}\right|<1\right\}$ and the total length of $\bigcup_{1 \leq j \leq k} \widetilde{M}_{r}^{j}$ is greater than $c_{3}$.

Choose $r_{1} \in\left(0, \rho_{1}\right)$ and a family of disjoint sets $\left\{M_{r_{1}}^{j}\right\}_{1 \leq j \leq j_{1}}$ such that $\bigcup_{1 \leq j \leq j_{1}} M_{r_{1}}^{j} \subset\left\{\left(x_{1}, x_{2}\right):\left|x_{1}\right|<\rho\right\}$ and the total length of $\bigcup_{1 \leq j \leq j_{1}} \widetilde{M}_{r_{1}}^{j}$ is greater than $c_{3} \rho$.

We proceed by induction. Let $\lambda_{1}$ be the Lebesgue measure of the set $[-\rho, \rho] \backslash$ $\bigcup_{1 \leq j \leq j_{1}} \widetilde{M}_{r_{1}}^{j}$. We find $r_{2} \in\left(0, r_{1}\right)$ and a family of sets $\left\{M_{r_{2}}^{j}\right\}_{1 \leq j \leq j_{2}}$ such that

(i) $\bigcup_{1 \leq j \leq j_{2}} M_{r_{2}}^{j} \subset\left\{\left(x_{1}, x_{2}\right):\left|x_{1}\right|<\rho\right\}$,

(ii) all sets in the family $\left\{M_{r_{k}}^{j}\right\}_{k=1,2 ; 1 \leq j \leq j_{k}}$ are disjoint, and

(iii) the total length of $\bigcup_{1 \leq j \leq j_{2}} \widetilde{M}_{r_{2}}^{j}$ is greater than $c_{3} \lambda_{1} / 2$.

The general inductive step is the following. Suppose that we have defined $r_{k}>0$ and families $\left\{M_{r_{k}}^{j}\right\}_{1 \leq j \leq j_{k}}$ for $k=1,2, \ldots, i$. Let $\lambda_{i}$ be the Lebesgue measure of the set $[-\rho, \rho] \backslash \bigcup_{1 \leq k \leq i} \bigcup_{1 \leq j \leq j_{k}} \widetilde{M}_{r_{k}}^{j}$. We find $r_{i+1} \in\left(0, r_{i}\right)$ and a family of sets $\left\{M_{r_{i+1}}^{j}\right\}_{1 \leq j \leq j_{i+1}}$ such that

(i) $\bigcup_{1 \leq j \leq j_{i+1}} M_{r_{i+1}}^{j} \subset\left\{\left(x_{1}, x_{2}\right):\left|x_{1}\right|<\rho\right\}$,

(ii) all sets in the family $\left\{M_{r_{k}}^{j}\right\}_{1 \leq k \leq i+1 ; 1 \leq j \leq j_{k}}$ are disjoint, and

(iii) the total length of $\bigcup_{1 \leq j \leq j_{i+1}} \widetilde{M}_{r_{i+1}}^{j}$ is greater than $c_{3} \lambda_{i} / 2$.

Let $T_{r}^{j}$ be the linear transformation that maps $M$ onto $M_{r}^{j}$ and let $N_{r}^{j}=T_{r}^{j}\left(N_{0}\right)$. Let $N_{*}=\bigcup_{k \geq 1} \bigcup_{1 \leq j \leq j_{k}} N_{r_{k}}^{j}$. It is easy to see that the Lebesgue measure of the set $[-\rho, \rho] \backslash \bigcup_{k \geq 1} \bigcup_{1 \leq j \leq j_{k}} \widetilde{M}_{r_{k}}^{j}$ is zero. It is also clear that $N_{*} \subset\left\{\left(x_{1}, x_{2}\right):\left|x_{1}\right| \leq \rho\right\}$.

Let $\widehat{D} \subset D_{\rho}$ be such that $\Lambda_{\rho}(\widehat{D}) \geq 4 \rho\left(1-\varepsilon_{0}\right)$ and for all $(x, \alpha) \in \widehat{D}$, we have $\left|K_{N_{0}}(x, \alpha)-K(x, \alpha)\right| \leq \varepsilon_{0}$. Let $\widehat{D}_{r}=\left\{(x, \alpha): \exists(y, \alpha) \in \widehat{D}\right.$ such that $\left.x=\left(r / r_{0}\right) y\right\}$. If $M_{r}^{j}=M_{r}+\left(b_{r}^{j}, 0\right)$, then we let $\widehat{D}_{r}^{j}=\{(x, \alpha): \exists(y, \alpha) \in \widehat{D}$ such that $x=y+$ $\left.\left(b_{r}^{j}, 0\right)\right\}$. Let $\Lambda_{r}^{j}$ be the measure $\Lambda$ restricted to $\left(-\left(r / r_{0}\right) \rho+b_{r}^{j},\left(r / r_{0}\right) \rho+b_{r}^{j}\right) \times(-\pi, 0)$. By the scaling and shift invariance of reflections, we see that for every $r>0$ and $j$ we have $\Lambda_{r}^{j}\left(\widehat{D}_{r}^{j}\right) \geq\left(r / r_{0}\right) 4 \rho\left(1-\varepsilon_{0}\right)$ and for all $(x, \alpha) \in \widehat{D}_{r}^{j}$, we have $\mid K_{N_{*}}(x, \alpha)-$ $K(x, \alpha) \mid \leq \varepsilon_{0}$. Let $D_{\rho}^{*}=D_{\rho} \backslash \bigcup_{k \geq 1} \bigcup_{1 \leq j \leq j_{k}} \widehat{D}_{r_{k}}^{j}$. Then $\Lambda_{\rho}\left(D_{\rho}^{*}\right)<4 \rho \varepsilon_{0}$, and for all $(x, \alpha) \in D_{\rho} \backslash D_{\rho}^{*}$, we have $\left|K_{N_{*}}(x, \alpha)-\bar{K}(x, \alpha)\right| \leq \varepsilon_{0}$. 
The set $N_{*}$ satisfies all assertions in the lemma except that it does not belong to $\mathcal{M}_{1}$ because it consists of an infinite number of curves (arcs of ellipses) and hence it does not satisfy condition (M2) of Section 2. To address this problem, we let $N=\bigcup_{1 \leq k \leq k_{0}} \bigcup_{1 \leq j \leq j_{k}} N_{r_{k}}^{j}$ for some $k_{0}<\infty$. It is easy to see that $N$ and the accompanying function $K_{N}$ satisfy all the conditions stated in the lemma if $k_{0}$ is sufficiently large.

Proof of Theorem 2.3. Recall the definition of a simple symmetric function from the beginning of Section 4. Suppose that $\widetilde{\mathbb{P}}$ satisfies (2.4). Recall that $\int_{-\pi}^{0} \sin \alpha d \alpha=-2$, let $m \geq 1$ be an integer and let $\gamma_{k}^{m}$ be defined by $-\int_{-\pi}^{\gamma_{k}^{m}} \sin \alpha d \alpha=k 2^{-m}$ for $k=0,1, \ldots, 2^{m+1}$. If we take $f(\alpha)=\mathbf{1}_{\left(\gamma_{n}^{m}, \gamma_{n+1}^{m}\right]}(\alpha)$ and $g(\alpha)=\mathbf{1}_{\left(\gamma_{j}^{m}, \gamma_{j+1}^{m}\right]}(\alpha)$, then (2.4) yields

$$
\int_{\left(\gamma_{j}^{m}, \gamma_{j+1}^{m}\right]} \int_{\left(\gamma_{n}^{m}, \gamma_{n+1}^{m}\right]} \widetilde{\mathbb{P}}(x, \alpha ; d \beta) \sin \alpha d \alpha=\int_{\left(\gamma_{n}^{m}, \gamma_{n+1}^{m}\right]} \int_{\left(\gamma_{j}^{m}, \gamma_{j+1}^{m}\right]} \widetilde{\mathbb{P}}(x, \alpha ; d \beta) \sin \alpha d \alpha .
$$

For a fixed integer $-\infty<k<\infty$, let

$$
a(n, j)=-\int_{[k / m,(k+1) / m]} \int_{\left(\gamma_{n}^{m}, \gamma_{n+1}^{m}\right]} \int_{\left(\gamma_{j}^{m}, \gamma_{j+1}^{m}\right]} \widetilde{\mathbb{P}}(x, \alpha ; d \beta) \sin \alpha d \alpha d x .
$$

It follows from (5.1) that $a(n, j)=a(j, n)$. Let $\beta_{n, 0}^{m}=\gamma_{n}^{m}$ and let $\beta_{n, i}^{m}$ be defined by

$$
-\int_{(k / m,(k+1) / m]} \int_{\gamma_{n}^{m}}^{\beta_{n, i}^{m}} \sin \alpha d \alpha d x=\sum_{0 \leq j \leq i-1} a(n, j)
$$

for $i=1, \ldots, 2^{m+1}$. Note that $\int_{(-\pi, 0]} \widetilde{\mathbb{P}}(x, \alpha ; d \beta)=1$ so

$$
\sum_{0 \leq j \leq 2^{m+1}-1} a(n, j)=-\int_{[k / m,(k+1) / m]} \int_{\left(\gamma_{n}^{m}, \gamma_{n+1}^{m}\right]} \sin \alpha d \alpha d x
$$

and, therefore, $\beta_{n, 2^{m+1}}^{m}=\gamma_{n+1}^{m}$.

Let $\widehat{\beta}_{n, i}^{m}=\left(\beta_{n, i}^{m}+\beta_{n, i+1}^{m}\right) / 2$ and $\widehat{K}_{m}(x, \alpha)=\left(x, \widehat{\beta}_{n, j}^{m}\right)$ for $(x, \alpha) \in(k / m,(k+$ $1) / m] \times\left(\beta_{j, n}^{m}, \beta_{j, n+1}^{m}\right]$. Since $a(n, j)=a(j, n)$, we have

$$
\int_{\left(\beta_{j, n}^{m}, \beta_{j, n+1}^{m}\right)} \sin \alpha d \alpha=\int_{\left(\beta_{n, j}^{m}, \beta_{n, j+1}^{m}\right)} \sin \alpha d \alpha .
$$

This shows that $\widehat{K}_{m}(x, \alpha)$ is a simple symmetric function according to Definition 4.1 .

Recall that for $K: D \rightarrow D$, the kernel $\mathbb{P}_{K}$ is defined by

$$
\mathbb{P}_{K}(x, \alpha ; d y, d \beta)=\delta_{K(x, \alpha)}(y, \beta) d y d \beta .
$$

It is elementary to check that $\mathbb{P}_{\widehat{K}_{n}}(x, \alpha ; d y, d \beta) \rightarrow \delta_{x}(y) d y \widetilde{\mathbb{P}}(x, \alpha ; d \beta)$ weakly on $D$ as $n \rightarrow \infty$. Note that we do not claim that $\widehat{K}_{n}$ arise as functions associated to reflecting sets $M \in \mathcal{M}_{2}$.

To prove Theorem 2.3, it will suffice to show that there exists a sequence of sets $M_{n} \in \mathcal{M}_{2}, n \geq 1$, satisfying conditions (a) and (b) of Theorem 2.3 and such that if the functions $K_{n}: D \rightarrow D$ correspond to $M_{n}$ 's, then $K_{n}-\widehat{K}_{n} \rightarrow 0$ pointwise. 
Fix some $\widehat{K}_{n}$ and let $\left(x_{1}^{k}, x_{2}^{k}\right)$ be one of the corresponding intervals as in Definition 4.1. Let $D_{k}=\left(x_{1}^{k}, x_{2}^{k}\right) \times(-\pi, 0)$. According to Lemma 5.1 and its proof, there exist a compact set $N_{k} \in \mathcal{M}_{1}$ and a set $D_{k}^{*} \subset D_{k}$ such that $\Lambda\left(D_{k}^{*}\right)<\varepsilon_{0} 2^{-k}$, and the following assertions hold:

(i) The function $K_{N_{k}}(x, \alpha)$ corresponding to $N_{k}$ is defined on $D_{k} \backslash D_{k}^{*}$.

(ii) For all $(x, \alpha) \in D_{k} \backslash D_{k}^{*}$, we have $\left|K_{N_{k}}(x, \alpha)-\widehat{K}_{n}(x, \alpha)\right| \leq \varepsilon_{0}$, and the light ray with the starting position and direction represented by $(x, \alpha)$ reflects exactly twice from $N_{k}$ before returning to $L_{*}$.

(iii) $N_{k} \subset\left(x_{1}^{k}, x_{2}^{k}\right) \times(-1 / n, 0)$.

Note that for $j \neq k$, either the sets $N_{j}$ and $N_{k}$ do not intersect or they are identical. Moreover, the construction presented in Lemma 5.1 allows us to assume that light rays that start in $D_{k} \backslash D_{k}^{*}$ do not leave $\left(x_{1}^{k}, x_{2}^{k}\right) \times(-\infty, 0)$. In this sense, the families of mirrors $N_{k}$ do not interfere with one another. Let $M_{n}^{*}=\bigcup_{k \geq 1} N_{k}$ and let $K_{n}^{*}(x, \alpha)$ be the function on $D$ corresponding to $M_{n}^{*}$. Let $D^{*}=\bigcup_{k \geq 1} D_{k}^{*}$ and note that $\Lambda\left(D^{*}\right) \leq \varepsilon_{0}$. We have the following:

(i) The function $K_{n}^{*}(x, \alpha)$ is defined on $D \backslash D^{*}$.

(ii) For all $(x, \alpha) \in D \backslash D^{*}$, we have $\left|K_{n}^{*}(x, \alpha)-\widehat{K}_{n}(x, \alpha)\right| \leq \varepsilon_{0}$, and the light ray with the starting position and direction represented by $(x, \alpha)$ reflects exactly twice from $M_{n}^{*}$ before returning to $L_{*}$.

(iii) $M_{n}^{*} \subset \mathbb{R} \times(-1 / n, 0)$.

It is easy to see that $M_{n}^{*} \in \mathcal{M}_{1}$ for every $n$, but it is not necessarily true (actually unlikely) that $M_{n}^{*} \in \mathcal{M}_{2}$. We can instead consider sets

$$
M_{n}=M_{n}^{*} \cup\left\{\left(x_{1}, x_{2}\right): x_{2}=-1 / n\right\} \cup \bigcup_{k}\left\{\left(x_{1}, x_{2}\right): x_{1}=x_{1}^{k} \text { or } x_{2}^{k},-1 / n \leq x_{2} \leq 0\right\} .
$$

It is easy to see that functions $K_{n}$ corresponding to $M_{n}$ satisfy all properties (i)(iii) listed above. Moreover, $M_{n}$ satisfy condition (M5) of Section 2 so $M_{n} \in \mathcal{M}_{2}$, according to Proposition 2.1. This completes the proof of the theorem.

\section{ACKNOWLEDGMENT}

The authors are grateful to Persi Diaconis and John Sylvester for their most helpful advice. The authors thank the anonymous referee for many suggestions for improvement.

\section{REFERENCES}

[1] K. Burdzy, R. Bass, Z. Chen and M. Hairer, Stationary distributions for diffusions with inert drift. Probab. Theory Rel. Fields 146 (2010) 1-47. MR2550357(2011b:60226)

[2] S. Chandrasekhar, Radiative transfer. Dover Publications, Inc., New York, 1960. MR0111583 $(22: 2446)$

[3] N. Chernov and R. Markarian, Chaotic billiards. Mathematical Surveys and Monographs, 127. American Mathematical Society, Providence, RI, 2006. MR2229799 (2007f:37050)

[4] F. Comets and S. Popov, Ballistic regime for random walks in random environment with unbounded jumps and Knudsen billiards. Ann. Inst. H. Poincaré Probab. Statist. (2012) (to appear).

[5] F. Comets, S. Popov, G. Schütz and M. Vachkovskaia, Billiards in a general domain with random reflections. Arch. Rat. Mech. Anal. 191, (2009) 497-537. MR2481068(2010h:37077)

[6] F. Comets, S. Popov, G. Schütz and M. Vachkovskaia, Erratum: Billiards in a general domain with random reflections. Arch. Ration. Mech. Anal. 193, (2009), 737-738. MR2525117 (2010h:37078) 
[7] F. Comets, S. Popov, G. Schütz and M. Vachkovskaia, Quenched invariance principle for the Knudsen stochastic billiard in a random tube Ann. Probab. 38 (2010) 1019-1061. MR 2674993 (2012c:60244)

[8] F. Comets, S. Popov, G. Schütz and M. Vachkovskaia, Knudsen gas in a finite random tube: transport diffusion and first passage properties. J. Stat. Phys. 140 (2010) 948-984. MR.2673342 (2012b:60317)

[9] S. Evans, Stochastic billiards on general tables. Ann. Appl. Probab. 11, 419-437 (2001). MR.1843052 (2002f:60134)

[10] K. Falconer, Fractal geometry. Mathematical foundations and applications. Second edition. John Wiley \& Sons, Inc., Hoboken, NJ, 1990. MR1102677 (92j:28008)

[11] R. Feres, Random walks derived from billiards. Dynamics, ergodic theory, and geometry, 179222, Math. Sci. Res. Inst. Publ., 54, Cambridge Univ. Press, Cambridge, 2007. MR2369447 (2009c:37033)

[12] R. Feres and H.-K. Zhang, The spectrum of the billiard Laplacian of a family of random billiards. J. Stat. Phys. 141, (2010) 1039-1054. MR2740402 (2011h:37052)

[13] R. Klette, K. Schlüns and A. Koschan, Computer vision. Three-dimensional data from images. Springer-Verlag Singapore, Singapore, 1998. MR1729491 (2000j:68190)

[14] S. Lalley and H. Robbins, Stochastic search in a convex region. Probab. Theory Relat. Fields 77, 99-116 (1988). MR921821 (89f:90078)

[15] M. Lapidus and R. Niemeyer, Towards the Koch snowflake fractal billiard: computer experiments and mathematical conjectures. Gems in experimental mathematics, 231-263, Contemp. Math., 517, Amer. Math. Soc., Providence, RI, 2010. MR2731085 (2012b:37101)

[16] M. Lapidus and R. Niemeyer, Families of Periodic Orbits of the Koch Snowflake Fractal Billiard. arXiv: 1105.0737

[17] S. Tabachnikov, Geometry and billiards. Student Mathematical Library, 30. American Mathematical Society, Providence, RI; Mathematics Advanced Study Semesters, University Park, PA, 2005. MR2168892 (2006h:51001)

[18] Wikipedia, Retroreflector, http://en.wikipedia.org/wiki/Retroreflector Online; accessed 4-March-2012.

[19] Wikipedia, Scotchlite, http://en.wikipedia.org/wiki/Scotchlite Online; accessed 4March-2012.

Department of Mathematics, University of British Columbia, 121 - 1984 Mathematics Road, Vancouver, British Columbia, Canada V6T $1 \mathrm{Z2}$

E-mail address: angel@math.ubc.ca

Department of Mathematics, Box 354350, University of Washington, Seattle, WashINGTON 98195

E-mail address: burdzy@math.washington.edu

Department of Mathematics, Massachusetts Institute of Technology, 2-180, 77 MasSachusetts Avenue, Cambridge, Massachusetts 02139

E-mail address: sheffield@math.mit.edu 